Juillet 2015

“Taux d'actualisation et rémunération du Capital"”

Christian GOLLIER 


\title{
Taux d'actualisation et rémunération du capital
}

\author{
Christian Gollier \\ Toulouse School of Economics
}

Juillet 2015

Plusieurs pays, dont la France et la Grande-Bretagne, ont adopté une méthode d'évaluation des investissements publics dans laquelle les taux d'actualisation peuvent s’écarter substantiellement des taux de rémunération du capital observés sur les marchés financiers. Les économistes s'accordent en effet pour reconnaître que les prix des actifs financiers n’offrent pas de bons signaux aux agents économiques pour orienter leurs choix d’investissements. Pour éviter le courttermisme qu'impliquent ces prix de marché, les Etats utilisent des taux d'actualisation réduits pour évaluer leurs investissements, plus proches de ceux utilisés par les parties prenantes qui les financent (contribuables, usagers,...).

Dans de nombreux secteurs de l'économie, les biens et services publics sont produits par des opérateurs parapublics ou privés que l'Etat rémunère pour leur permettre de couvrir leurs coûts et de rémunérer leurs risques. La question se pose dès lors de la rémunération du capital de ces opérateurs. Comme la théorie économique enseigne que dans un monde parfait, le coût du capital est égal au taux d’actualisation, plusieurs autorités de régulation de ces marchés publics ont récemment proposé d'utiliser ces taux d'actualisation réduits comme référence pour fixer cette rémunération du capital offerte par l'Etat à ces opérateurs. Cette note a pour objectif de montrer que cette proposition conduirait à des conséquences diamétralement opposées à celles voulues par les auteurs des rapports Lebègue, Gollier et Quinet qui ont argumenté en faveur de ces taux d’actualisation réduits. 


\section{Le cas des marchés parfaits}

Dans un monde parfait, les prix de marché offrent les bons signaux qui permettent aux agents économiques de prendre des décisions compatibles avec l'intérêt général. Par exemple, le taux de rendement observé sur les marchés financiers pour les actifs sans risque est le taux d'actualisation que doivent utiliser les entrepreneurs pour évaluer leur projet d'investissement sans risque. Comme ce taux d'intérêt est aussi le coût d'opportunité de leur capital, c'est-à-dire leur Coût Moyen Pondéré du Capital (CMPC) ${ }^{1}$ marginal, ils seront naturellement amenés à utiliser ce taux pour actualiser les flux futurs de leur projet pour en déterminer la valeur. Dans ce cadre valeur sociale et valeur privée sont parfaitement identiques. Un opérateur délivrant un service ou un produit dans le cadre d'une commande publique devrait voir son capital rémunéré au taux d'intérêt du marché si cette activité est sans risque. Ce taux d'actualisation sera à la fois égal à la productivité marginale du capital sans risque dans l'économie qu'au taux marginal de substitution entre les consommations présente et future. Ceci assure que l'investissement du dernier euro dans ce projet sans risque n'affecte pas le bien-être collectif à la marge, qu'il soit financé par une réallocation du capital dans l'économie, ou par une augmentation de l'épargne collective.

Le caractère risqué du projet d'investissement considéré ne change pas le message, à l'exception du fait que des projets dont la réalisation génère une plus forte augmentation du risque agrégé nécessiteront un rendement espéré plus élevé pour attirer les capitaux, parce que les agents économiques éprouvent de l'aversion au risque. Dans ce cas aussi, CMPC marginal, taux de rendement espéré des actifs et taux d'actualisation seront identiques, mais supérieurs au taux d'intérêt sans risque.

Dans un tel contexte, il est donc toujours désirable d'offrir une rémunération du capital des opérateurs de services publics qui soit égale au taux d’actualisation utilisé pour évaluer leurs investissements.

\section{Rôle du taux d'actualisation public dans un monde imparfait}

\footnotetext{
${ }^{1}$ Le Coût Moyen Pondéré du Capital est composé d'une part du coût des fonds propres et d'autre part du coût de la dette. Il est le fruit de la pondération de ces deux éléments en fonction de leur poids relatif dans le financement d'une entreprise ou d'un projet.
} 
Mais le monde est imparfait. En particulier, les marchés financiers sont intrinsèquement inefficaces. Beaucoup d'investisseurs sont myopes dans le sens où ils ont tendance à souspondérer dans leur décision l'impact des conséquences à long terme de ces décisions sur leur propre bien-être. Les sciences de la cognition et la psycho-économie nous enseignent aussi qu'ils sont souvent affectés par l'incohérence intertemporelle de leur choix, de manière à les conduire à refuser d'intégrer dans leur processus cognitif les informations sur les risques encourus, même quand ces informations sont publiques et gratuites. Par ailleurs, parce que la plupart n'ont pas eu personnellement à affronter d'événements financiers rares, les croyances des investisseurs n'intègrent pas ces risques extrêmes alors qu'ils font partie de l'univers des scenarii possibles. De plus, l'existence de frictions importantes sur les marchés financiers empêche la réalisation d'échange mutuellement avantageux. Par exemple, beaucoup d'agents économiques sont dans l'incapacité d'emprunter à court terme pour affronter des conditions financières temporaires adverses, même si leur perspective de long terme est prospère. Ce problème d'illiquidité et d’inefficacité du marché du crédit empêche les agents économiques de lisser leurs chocs financiers dans le temps, augmente artificiellement leur aversion au risque et réduit leur horizon temporel.

Le choix du taux d'actualisation contient aussi une dimension éthique importante. Une part importante des investissements mis en œuvre aujourd'hui bénéficiera aux générations futures qui n'ont pas la possibilité aujourd'hui d'exprimer leurs préférences, notamment à travers des transactions sur les marchés financiers. En conséquence, les prix des actifs financiers ont tendance à ne refléter que les préférences des générations présentes, dont l'altruisme intergénérationnel est limité. Hors, les préférences collectives se doivent de représenter de façon équitable l'intérêt commun de toutes les générations, présentes et à venir. Tous ces arguments justifient qu'on n’accorde pas aux prix de marché l'aura que la théorie économique classique leur a donnée pour décentraliser une allocation efficace de nos ressources rares.

Sans rentrer dans les détails, la démarche commune suivie dans les rapports Lebègue, Gollier et Quinet (ainsi que de nombreux rapports écrits à l'étranger sur le même sujet) consiste à définir une fonction de bien-être collectif (intergénérationnel) et de dériver de cette fonction un critère d'évaluation des investissements tel que seuls les projets qui augmentent ce bien-être collectif 
passeront positivement ce critère. La représentation de cet intérêt général communément acceptée par les philosophes et les économistes est basée sur le modèle d'espérance d'utilité. Il conduit à un critère d'évaluation des investissements de la Valeur Actualisée Nette (VAN) qualitativement identique à celui utilisé depuis plus d'un siècle par les particuliers, les entreprises et les Etats. Néanmoins, les taux d'actualisation qu'il faut utiliser dans ce calcul de VAN socio-économique peuvent effectivement s’écarter considérablement des taux d’actualisation dérivés des prix observés sur les marchés financiers et que les agents économiques sont incités à utiliser pour maximiser la valeur de marché de leur activité. Valeur sociale et valeur de marché deviennent deux choses divergentes dans ce monde imparfait.

Le rapport Quinet dérive de cette méthodologie un système d'actualisation basé sur la formule du MEDAF, où le taux (réel) d'actualisation $r$ d'un projet spécifique identifié par son $\beta$ est défini par l’équation suivante :

$$
r=r_{f}+\beta \pi
$$

où $r_{f}$ est le taux sans risque et $\pi$ est la prime de risque. Le rapport Quinet quantifie de plus ces deux paramètres en fonction de la maturité des cash-flows à évaluer :

$$
\begin{array}{ccc}
\text { Court terme: } & r_{f}=2.5 \% & \pi=1.5 \% \\
\text { Long terme: } & r_{f}=1.5 \% & \pi=3.0 \%
\end{array}
$$

L'estimation de ces paramètres reste un sujet de débat parmi les experts, comme on peut le voir par exemple dans les divergences constatées entre les rapports Quinet et Gollier pour le choix de ces paramètres. Le rapport Gollier recommandait un taux sans risque réel autour de $2 \%$, et une prime de risque de 3\%. Ces estimations sont fondées sur une calibration spécifique des paramètres de préférence pure pour le présent et d'aversion aux inégalités, ainsi que sur des représentations divergentes des scénarii probabilisés de la croissance économique future. Les rapports cités plus haut insistent tous sur la nécessité de réévaluer périodiquement les paramètres $\left(r_{f}, \pi\right)$ utilisés pour évaluer les cash-flows de court terme, en fonction des changements d'anticipation de croissance économique à court et moyen terme. Par contre, les paramètres $\left(r_{f}, \pi\right)$ à utiliser pour évaluer les cash-flows longs sont par nature plus stables. Ils ne devraient 
être modifiés qu'en cas de changement conséquent des anticipations de croissance de long terme de l’économie française et européenne.

\section{Quelle rémunération du capital des opérateurs de service public ?}

Les rapports Lebègue, Gollier et Quinet n’avaient pas vocation à discuter de la rémunération du capital à offrir aux opérateurs en charge de la production de biens et services en délégation de service public, tels qu'ils existent dans les secteurs du transport, de l'énergie ou des télécommunications par exemple. Ainsi, dans sa lettre de saisine du 11 mai 2009 créant la Commission Gollier au Conseil d’Analyse Stratégique, ${ }^{2}$ le Ministre Borloo charge cette commission de «déterminer les avancées théoriques actuelles les plus applicables dans l'évaluation des décisions et projets publics. Le but est de faciliter et d'harmoniser la mise en œuvre pratique des méthodes et des principes d'évaluation par les administrations [...]».

Néanmoins, le fait que dans un monde parfait (de premier rang), le taux d'actualisation socialement désirable soit égal au CMPC marginal, donc au taux d’intérêt du marché, pourrait suggérer que l'on pourrait utiliser les taux proposés dans ces rapports pour déterminer la juste rémunération du capital des opérateurs de service public. Il n’en n’est rien. En fait, ces rapports sont muets sur ce sujet, tout comme la littérature scientifique sur laquelle sont fondés ses rapports. $^{3}$ Dans la suite de cette note, je présente une critique de l'utilisation des taux d’actualisation socio-économique (2) pour rémunérer le capital des opérateurs.

Pour illustrer, imaginons un opérateur dont le capital est concentré sur un actif très long dont le $\beta$ est égal à 1. En suivant le rapport Quinet, le taux d'actualisation socio-économique réel pour cette entreprise devrait être égal à $1.5 \%+1 \times 3 \%=4.5 \%$. Supposons en même temps que les prix des actifs financiers actuels révèlent un taux d’intérêt réel sans risque de $2 \%$, et une prime de risque de 6\%, de sorte que si l'opérateur devait financer son capital par appel à des investisseurs privés, son CMPC réel serait de $2 \%+1 \times 6 \%=8 \%{ }^{4}{ }^{4}$ Il faut considérer plusieurs cas de figure.

Considérons d'abord le cas d'un opérateur entièrement détenu et contrôlé par l'Etat omniscient et omniprésent. Dans ce cas, le problème de la rémunération de son capital ne constitue qu'un jeu

\footnotetext{
${ }^{2}$ Voir Gollier (2011), Annexe 1.

${ }^{3}$ Dans cette note, j'évite de revenir sur les arguments scientifiques qui fondent les recommandations de cette littérature. Pour une synthèse récente, voir Gollier (2012) et IPCC (2014, chapitre 3).

${ }^{4}$ Ce raisonnement est fondé, pour simplifier, sur l'hypothèse que le projet est financé uniquement par des fonds propres mais serait également valable pour un projet financé en partie par de la dette.
} 
d'écritures comptables sans implication réelle. Il suffit à l'Etat d'imposer à son opérateur de réaliser tous les projets d'investissement dont le rendement espéré est supérieur à 4.5\%. Tous les investissements dont la rentabilité espérée est comprise entre 4.5\% et le taux du marché de $8 \%$ se traduiront immédiatement en moins-values dans le bilan de l'opérateur, bien que ces projets créent de la valeur socioéconomique positive. Cette perte sèche pour l’opérateur est épongée par l'Etat, d'une façon ou d'une autre (par des subventions pas exemple).

Mais l'Etat n'est pas omniscient, ce qui l'oblige à déléguer certaines décisions d'investissements à l'opérateur. Quel que soit le statut de ce dernier, ceci pose un problème d'agence. Si l'Etat impose une rémunération du capital de $4.5 \%$ à l'opérateur comme il est proposé, étudions les incitations que ce contrat génère. Si l'opérateur finance ses investissements par les marchés financiers, son coût du capital effectif est de 8\%. L’opérateur est donc incité à limiter au mieux ses investissements puisque ces derniers se font à perte. Le même résultat est obtenu si l'opérateur autofinance ces investissements. En effet, il existe dans l'économie des projets de même profil de risque dont la rémunération espérée est supérieure ou égale à 8\%. Confronté à ce coût d'opportunité du capital, une rémunération du capital de 4.5\% n’incite pas au réinvestissement au sein de l'entreprise puisque tout réinvestissement se traduirait par une rémunération du capital inférieure à son coût d'opportunité. Pour l'opérateur, ce réinvestissement conduit inéluctablement à une destruction de valeur. Dans tous les cas, l'opérateur supporte donc un coût supplémentaire à utiliser son capital pour produire ces biens et services publics, plutôt que de l'investir dans d'autres actifs ou dans d'autres activités économiques de même profil de risque. Si l'opérateur est indépendant la rémunération du capital fixée par le régulateur doit être égale à son coût d'opportunité tel que déterminé en référence au marché afin d'éviter toute désincitation à l’investissement.

Pour résumer, la fixation de la rémunération des fonds propres à un niveau inférieur à celui exigé par le marché pour des investissements ayant un profil de risque similaire- par exemple en le fixant au taux d'actualisation socioéconomique - réduit l'incitation à investir. Alors que la baisse du taux d'actualisation socioéconomique a vocation à renforcer l'investissement public, en particulier dans les projets de long terme peu risqués, une règle consistant à identifier la rémunération du capital des opérateurs à ce taux d'actualisation aura au contraire pour conséquence de réduire ces investissements. 
Si les travaux synthétisés dans les rapports Lebègue, Gollier et Quinet étaient de nature purement normative pour déterminer des règles d'évaluation des investissements compatibles avec l'intérêt général, la problématique de la fixation de la rémunération du capital des opérateurs est de nature beaucoup plus positive. Ce sont des approches fondamentalement différents. Il est erroné vouloir utiliser les résultats de l'un pour résoudre l'autre. Ces rapports n'ont juste rien à dire sur le sujet de la juste rémunération du capital. Dans un optimum de second rang, il y a une déconnection entre le calcul de l'allocation socialement désirable et le prix auquel on rémunère les opérateurs qui produisent les biens publics.

\section{Un problème de cohérence}

Comme on l'a vu dans les sections précédentes, deux logiques s'affrontent pour déterminer la juste rémunération du capital. Il y a d'une part la logique de marché, fondée sur le coût d'opportunité de ce capital pour les opérateurs, et il y a d'autre part la logique socioéconomique fondée sur une approche de bien-être. Si la logique socioéconomique a une légitimité incontestable pour déterminer des règles d'évaluation des investissements publics, elle n’a pas force de justification dans le cadre de la rémunération du capital dans un monde de second rang. Le pire serait encore de mélanger les deux logiques, par exemple en justifiant le taux sans risque $r_{f}$ par l'observation des taux d'intérêt réels sur les marchés, tout en justifiant la prime de risque $\pi$ sur la base de l’approche socioéconomique suivie dans les rapports Gollier et Quinet.

Pour comprendre les dangers du mélange des deux logiques, il faut rappeler les deux grands paradoxes de la théorie de la finance. ${ }^{5}$ Le paradoxe du taux d'intérêt sans risque provient du fait que le taux d'intérêt réel $r_{f}$ observé sur les marchés financiers au XXe siècle a été en moyenne très faible (autour de $1 \%$ aux USA, autour de $-1 \%$ en France). A de tels taux, les ménages auraient dû beaucoup moins épargner que ce qu’on a observé durant la même période, avec une accumulation phénoménale du capital. En d'autres termes, la logique de marché aurait conduit à une rémunération du capital sans risque beaucoup plus faible qu'en suivant la logique socioéconomique. Au contraire, le paradoxe de la prime de risque provient du fait que la prime de risque $\pi$ observée sur les marchés durant le siècle écoulé (autour de $6 \%$ aux USA) a été très

\footnotetext{
${ }^{5}$ Pour de bonnes synthèses, lire Kocherlakota (1996), Cochrane (2001) et Gollier (2001).
} 
élevée. Avec une telle prime de risque de marché, on aurait dû voir les ménages se déchainer pour investir dans les actifs les plus risqués dans l'économie, alors que dans la réalité la plupart d'entre eux ont investi leur épargne dans des actifs sans risque. En d'autres termes, la logique de marché aurait conduit à une rémunération du capital risqué beaucoup plus élevée qu'en suivant la logique socioéconomique. Dans un souci de cohérence, la recherche en théorie de la finance a d'ailleurs éprouvé beaucoup de difficultés à construire des modèles réalistes qui permettent de résoudre simultanément les deux paradoxes. ${ }^{6}$

On comprend donc dans ce contexte que laisser les agents économiques «faire leur marché » entre les deux logiques pour justifier leur prétention en termes de rémunération du capital ouvre la porte à toutes les distorsions possibles. Les opérateurs de projets peu risqués maximiseront leur demande de rémunération en favorisant la logique socioéconomique (qui recommande un $r_{f}$ plutôt élevé). Quant à eux, les opérateurs de projets très risqués favoriseront la logique de marché (qui conduit à un $\pi$ élevé). Plus généralement, on peut s'attendre à ce que les financeurs demandent un $r_{f}$ de marché et un $\pi$ socioéconomique, et l'inverse pour les opérateurs. Tout cela est incohérent et nécessite une clarification. Dans un monde de second rang où les marchés financiers n'agrègent qu'imparfaitement les préférences des individus qui le compose, les approches de marché et socio-économique sont distinctes et inconciliables, comme le montre les nombreux paradoxes de la théorie moderne de la finance. Les objectifs de ces deux approches sont différents et ne peuvent être mélangés dans un même raisonnement économique sans résulter en de dangereux contresens. L'approche de marché à vocation à déterminer la rémunération du capital pour un opérateur qui soit compatible avec une incitation efficace à investir, ce qui impose d'identifier cette rémunération au coût d'opportunité du capital observable sur les marchés financiers. L'approche socio-économique a pour vocation quant à elle de déterminer les choix d'investissements se traduisant par le plus grand bénéfice pour la société (au sens de bien-être collectif). L'approche socio-économique ne peut donc être utilisée pour déterminer la rémunération d'un opérateur.

\section{Conclusion}

\footnotetext{
${ }^{6}$ Voir Barro (2009) par exemple.
} 
Une littérature scientifique importante s’est développée durant ces deux dernières décennies pour recommander aux autorités publiques de réduire les taux d'actualisation qu'elles utilisent pour évaluer leurs investissements. Cette recommandation, compatible avec la volonté de construire une croissance économique plus soutenable, trouve ses fondements dans une théorie cohérente du choix social qui intègre les générations futures.

Récemment en France, des instances de régulation de certains marchés ont été conduits à modifier la rémunération du capital des opérateurs en charge de la production de certains biens publics. Elles justifient cette réduction sur la base de cette littérature et des rapports publics qui s'en sont fait l'écho, alors même que cette littérature et ces rapports n'adressent pas du tout cette question. En fait, la réduction de la rémunération du capital imposée par ces instances aura un impact exactement inverse à celui d'une réduction du taux d'actualisation. Elle conduira à une baisse des investissements justement là où la littérature récente suggère qu'il faudrait les accroître. Dans un monde de second rang dans lequel l'Etat est incapable d'agir sur les prix de marché, il est indispensable de déconnecter le problème de la fixation des bons signaux-prix pour évaluer une politique publique de celui de la rémunération des opérateurs en charge de sa mise en œuvre.

\section{Bibliographie}

Barro, R.J., (2009), Rare disasters, asset prices, and welfare costs, American Economic Review, 99, 243-264.

Cochrane, J., (2001), Asset pricing, Princeton University Press.

Gollier, C., (2001), The economics of risk and time, MIT Press.

Gollier, C., (2011), Le calcul du risque dans les investissements publics, Centre d'Analyse Stratégique, Rapports \& Documents n³6, La Documentation Française.

Gollier, C., (2012), Pricing the planet's future: The economics of discounting in an uncertain world, Princeton University Press. 
IPCC, (2014), Climate Change 2014: Mitigation of Climate Change, Contribution of Working Group III to the Fifth Assessment Report of the Intergovernmental Panel on Climate Change [Edenhofer, O., R. Pichs-Madruga, Y. Sokona, E. Farahani, S. Kadner, K. Seyboth, A. Adler, I. Baum, S. Brunner, P. Eickemeier, B. Kriemann, J. Savolainen, S. Schlömer, C. von Stechow, T. Zwickel and J.C. Minx (eds.)], Cambridge University Press, Cambridge, United Kingdom and New York, NY, USA.

Kocherlakota, N.R., (1996), The Equity Premium: It's Still a Puzzle, Journal of Economic Literature, 34, 42-71.

Lebègue, D., (2005), Révision du taux d'actualisation des investissements publics, Commissariat Général au Plan, Paris.

Quinet, E., (2013), L'évaluation socioéconomique des investissements publics, Commissariat Général à la Stratégie et à la Prospective, Rapports et Documents, Paris. 